\title{
论 文＼cjkstart紧致化视觉计算专题
}

\section{基于多尺度CRF朝向敏感性的形状特征表示方法}

\author{
段婷，李明 ${ }^{*}$, 沈辉, 胡德文 \\ 国防科技大学智能科学学院, 长沙 410073 \\ *E-mail: liming78@nudt.edu.cn \\ 收稿日期: 2021-06-01; 接受日期: 2021-11-04; 网络版发表日期: 2022-03-30 \\ 国家重点研发计划(编号: 2018YFB1305101)和国家自然科学基金(批准号: 62076248, 62036013)资助项目
}

\begin{abstract}
摘要 图像中物体的轮廓是稳定的, 是旋转、平移和尺度等不变性识别的基础. 本文对初级视皮层的神经元细胞 进行建模, 借鉴神经元方位选择性提取图片朝向信息, 实现更接近大脑感知的形状特征表示策略. 首先, 以线段为 基元表征物体的几何特征, 引入朝向强度与尺度空间来获取更丰富的轮廓信息; 其次, 利用神经编码稀疏性指标, 改进朝向关系匹配方法和尺度融合策略, 获得了表示能力更强的图像形状特征. 实验表明, 基于该特征表示策略 的形状检索算法实现了更高的检索正确率, 在存在遮挡和噪声干扰情况下, 本文方法表现出更好的鲁棒性.
\end{abstract}

关键词形状特征表示, 多尺度融合, 方位选择性, 几何特征提取, 图像表征

\section{1 引言}

在计算机视觉领域，现有的深度神经网络具有计 算量大、占用内存多、可解释性差等缺点，压缩模型 大小和减少网络深度是目前的解决途径之一 ${ }^{[1]}$. 有研 究者借助生理视觉原理, 对目标图像预先进行特征抽 取和表示, 显著减少了深层网络的规模, 降低了数据 的维度 ${ }^{[2]}$. 因此采用高效的特征表示方法是实现紧致 化视觉的重要途径之一. 由于在变化的环境或姿态下, 图像的几何特征是恒定的，提取并利用图像稳定的形 状特征对提高视觉任务的鲁棒性有帮助.

现有的特征提取方法如形状上下文(shape context, $\mathrm{SC})^{[3]}$ 、三角形区域表示(triangle-area representation, $\mathrm{TAR})^{[4]}$ 、距离集(distance sets) ${ }^{[5]}$ 、傅里叶描述子(Fourier descriptor, $\mathrm{FD})^{[6]} 、 \mathrm{Hu}^{\text {矩 }}{ }^{[7]}$ 、Zernike矩 ${ }^{[8]}$ 等, 均使用
轮廓点的分布关系表征形状, 后续如内距离形状上下 文(inner-distance shape context, IDSC) ${ }^{[9]}$ 和高度函数 (height function, $\mathrm{HF})^{[10]}$ 等算法在 $\mathrm{SC}$ 的基础上做了改 进, 但都需要匹配像素点对, 这将增加计算代价. 认知 学研究发现, 人脑的识别过程更关注形状的方位、曲 率等中高级特征 ${ }^{[11]}$, 将形状的中高级特征映射成二值 编码, 用编码的距离度量相似度, 会使形状的匹配更 加高效, 且可解释性强.

基于中高级特征编码的形状描述子如词袋(bag of

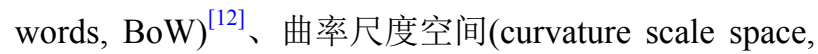
$\mathrm{CSS})^{[13]}$ 以及编码角度 ${ }^{[14]}$ 和轮廓段特征 ${ }^{[15]}$, 几何表示更 加明确, 具有更好的可解释性. 但是上述方法在学习能 力、计算时间、空间能耗上仍不能与人视觉系统的优 异识别性能相比, 并且特征反映的目标形状与人的直 观感受不完全一致, 编码特征的描述子也无法迁移到

引用格式: 段婷, 李明, 沈辉, 等. 基于多尺度 $\mathrm{CRF}$ 朝向敏感性的形状特征表示方法. 中国科学: 技术科学, 2022, 52: 682-688 Duan T, Li M, Shen H, et al. Shape feature representation by the multiscale model of CRF orientation selectivity (in Chinese). Sci Sin Tech, 2022, 52: 682-688, doi: 10.1360/SST-2021-0226 
仿生视觉的不同几何特征空间中.

超柱模型 ${ }^{[16]}$ 借鉴视觉神经系统的优异性能，模拟 哺乳动物初级视皮层对朝向信息的选择性反应机制来 提取和表示图像中的线性或近似线性特征，提高了图 像匹配的效率. 但受限于缺少有效的多特征融合方法, 现有超柱模型均未考虑朝向的强度和尺度信息，故而 无法表示丰富的轮廓信息.

本文基于超柱模型及其代表性算法线条上下文 (line context, LC $)^{[16,17]}$, 通过模拟视觉神经编码的稀疏 现象, 克服引入朝向强度之后的信息冗余问题, 并利用 朝向稀疏性指标预测最优尺度，实现多尺度最优融合， 得到了多尺度形状特征的低维描述. 实验结果表明, 该 特征表示方法提升了模型获取特征的能力，能够应用 于较小的训练集, 尤其在存在严重遮挡及噪声干扰情 况下表现出优良的特征抽取能力.

\section{2 经典感受野朝向敏感模型}

为了提高超柱模型用于特征提取的效果，本文提 出多尺度融合的线条上下文算法，实现方案为：首先 对输入检测图像建立多级尺度空间金字塔，用朝向柱 模型依次遍历金字塔中的图像，并引入朝向强度来提 取图像轮廓中的直线段与近似直线段. 再利用空间金 字塔对图像中的朝向信息进行多尺度的特征提取，分 别通过传统融合与最优融合两种策略将多尺度的特征 信息相统一. 最后基于闵可夫斯基距离进行相似度的 度量, 得到最终图像的匹配结果.

\section{1 超柱模型与朝向强度}

首先介绍本文中使用的模型. 借鉴初级视皮层中 简单细胞的聚类方式，模型中使用可以紧凑组合起来 的六边形代表简单细胞. 根据简单细胞的朝向敏感性, 使用朝向片 $b_{1} \sim b_{n}$ 表示一个朝向柱内 $n$ 个具有敏感朝向 的神经元. 图 1 为 $b_{1} \sim b_{19}$ 的朝向柱模型. 每个朝向柱内的 神经元的朝向角度范围是 $0^{\circ} \sim 180^{\circ}$, 因此每个朝向柱可 以表示 $n$ 个间隔为 $180^{\circ} / n$ 的子区间. 在模型上使用局部 梯度方向直方图Hog算子识别感受野内线条的朝向信 息, 得到感受野内所有兴奋型神经元的激活强度、激 活朝向和空间位置 $\left(I o A, \alpha, R F_{N}, R F_{n}\right)$, 其中 $I o A$ 代表激 活强度(intensity of activation), $\alpha$ 代表激活朝向, $R F_{N}$ 和 $R F_{n}$ 分别为超柱模型中朝向柱和朝向片的编码. 研究发
现在视觉空间中央 $10^{\circ}$ 内的位置刺激会引发一个几乎 恒定大小的宽神经活动带 ${ }^{[18]}$, 因此本文使用的朝向间 隔与超柱模型 ${ }^{[16]}$ 保持一致，设置 $n=19$; 由于人类大脑 皮层中“兴奋型-抑制型”突触的比例大约为 $9: 1$, 为体 现这种视觉稀疏性, 本模型中的兴奋型神经元的数量 设为 $1 \sim 3$, 对应于激活的强度, 将所有的兴奋型神经元 的信息整合成序列作为朝向柱阵列的输出. 图 1 为朝向 柱中不同朝向强度的激活线段示意, $L_{1} \sim L_{3}$ 的朝向强度 依次增强, 各个线段基元代表的含义如表1所示.

根据初级视皮层简单细胞互拮抗感受野对拮抗边 缘的敏感, 构建不同最优朝向的拮抗感受野模型. 首先 朝向柱感受野可以感知视野范围内的 $n$ 个敏感朝向, 将 $N$ 个朝向柱的感受野 $\left(A_{1} \sim A_{n}\right)$ 相互连接代表 1 个朝向柱 阵列的感受野. 其中 $N \times n$ 个神经元的感受野构成经典 感受野(classical receptive field, CRF). 图2为一个朝向 柱阵列的感受野, 依次由 $1,6,12,18$ 个 $9 \times 9$ 的像素区域, 共4层感受野拼接而成, 为了不使图像信息遗漏, 采用 重叠的方式组合.

\section{2 形状匹配与相似性度量}

为了获取更多的局部信息，在超柱模型朝向关系 的计算基础上扩大激活朝向片之间的计算范围，增加

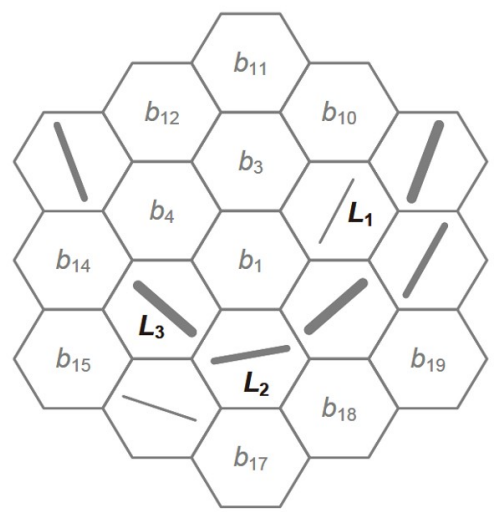

图 1 不同线段在朝向柱内的激活强度

Figure 1 The activation of an orientation column for different segments.

表 1 图1中各个线段代表的不同信息

Table 1 Parameter values of the segments in Figure 1

\begin{tabular}{cccc}
\hline 参数 & $L_{1}$ & $L_{2}$ & $L_{3}$ \\
\hline$I o A$ & 1 & 2 & 3 \\
$\alpha$ & $7 \Delta \theta$ & $\Delta \theta$ & $15 \Delta \theta$ \\
{$\left[R F_{N}, R F_{n}\right]$} & {$[N, 2]$} & {$[N, 6]$} & {$[N, 5]$} \\
\hline
\end{tabular}




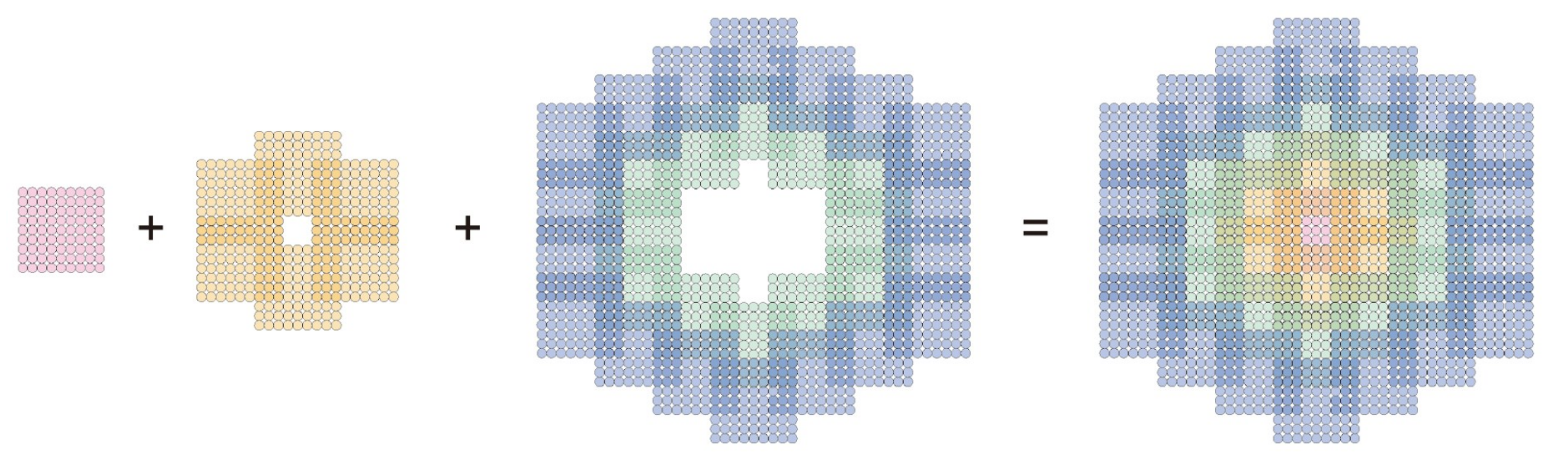

图 2 朝向柱组成感受野示意图

Figure 2 Illustration for constructing the receptive field by orientation column arrays.

朝向柱的层数，计算激活朝向片 6 层范围内的bin关 系 $^{[16]}$. 在bin的相似度度量过程中，引入闵可夫斯基距 离( $\mathrm{L} p$ 范数):

$\operatorname{sim}(a, b)=\sqrt[p]{\sum_{i=1}^{N}\left(|a(i)-b(i)|^{p}\right)}$.

相似度的计算过程依据下式:

$\operatorname{sim}(a, b)= \begin{cases}1-\frac{\sqrt[p]{\sum_{i=1}^{19} d i(i)}}{19}, & a \cup b \neq\{0\}, \\ 1, & a \cap b=\{0\}, \\ 0, & \text { 其他, }\end{cases}$

其中,

$\operatorname{diff}(i)= \begin{cases}H^{p}, & a \oplus b=0, \\ |a(i)-b(i)|^{p}, & a \oplus b=1 .\end{cases}$

实验中取 $p=2$, 抑制因子 $H=19 / 2 \approx 10$. 改进后增大了同 类形状间的相似度差异, 进一步提高了模型的鲁棒性.

\section{3 多尺度的融合策略}

使用不同尺度的超柱模型可以提取图像形状更准 确全面的特征, 但如何融合这些多尺度特征, 尤其是篮 选最适合的尺度结果, 一直缺乏可靠策略. 有研究者借 鉴人类视觉对不同尺度形状的敏感性，提出依赖于敏 感曲线的加权平均融合策略 ${ }^{[19]}$ (下称“经典尺度融合策 略”). 但经典尺度融合策略对于所有目标图像使用相 同的敏感曲线(权重曲线), 未考虑目标图像的差异, 更 无法预测和筛选最适合目标图像的尺度参数.

本文根据最优尺度与目标图像复杂度相关的事
实 ${ }^{[20]}$, 使用朝向矩阵稀疏性 ${ }^{[21]}$ 对各尺度与目标图像的 符合程度进行预测, 篮选出若干个最优尺度, 然后再利 用敏感曲线进行特征融合(下称“最优尺度融合策略”).

为了验证最优尺度预测和融合的效果, 本文对比 采用了此两种融合策略.

（1）经典尺度融合策略

人眼在辨别图像时, 图像的整体轮廓特征一般在 远距离观察时获取, 而近距离观察主要获取的则为纹 理等局部细节特征，这两种情况分别对应图像的宏观 尺度和微观尺度. 基于人眼视觉的上述特征, 在多尺 度融合时, 宏观尺度下, 对应的权重应偏大, 微观尺度 应使用小的权重 ${ }^{[19]}$.

一般情况下, 选择图像的原始尺度(尺度标号记为 $n=1$ ) 为最宏观尺度, 其余尺度通过逐级放大图像得到 (标号分别记为 $n=2,3, \ldots, N$ ). 不同尺度的融合权重随 着标号的增加线性递减:

$\mathbf{w}=\left(\omega_{1}, \ldots, \omega_{n}, \ldots, \omega_{N}\right)$,

$\omega_{n}=(N-n+1) / \sum_{i=1}^{N} i$

其中, $\mathbf{w}$ 是所有尺度的权重组成的行向量, 上述公式描 述的权重曲线如图3(a)所示.

在缺乏与最优尺度相关的先验知识时, 经典融合 策略下的检索结果会优于多尺度平均的检索结果.

(2) 最优尺度融合策略

经典尺度融合策略对于所有图像使用相同的权重 曲线, 并且认为所有图像的最具鉴别力的尺度(下称最 优尺度, 标号记为 $n_{\mathrm{opt}}$ ) 为原始尺度, 即假设最优尺度与 原始尺度一致 $\left(n_{\mathrm{opt}}=n_{1}\right)$. 

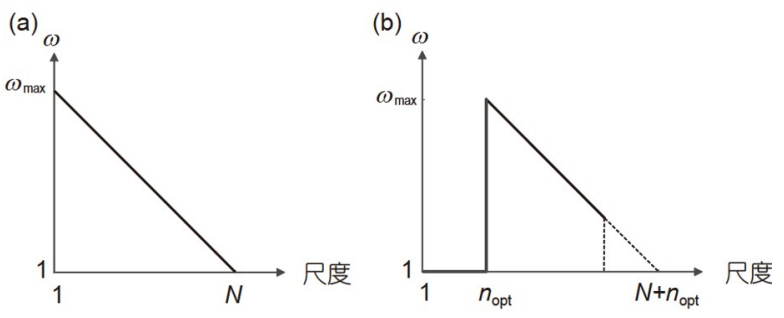

图 3 多尺度融合策略的尺度权重

Figure 3 Weight curves in multi-scale fusion strategy.

但该假设并不总是成立. 图像内容的复杂度对图 像表征能力最优的尺度有显著影响 ${ }^{[22]}$. 不同图像在各 自原始尺度下的复杂程度各不相同，所以他们的最优 尺度一般也不相同. 比如有的图形过于简单，朝向柱 阵列在原始尺度下提取到的特征矩阵过于稀疏，无法 获取足够特征来表示这些图像. 此类图像与朝向柱阵 列的感受野大小不匹配，需要将图像放大或者感受野 缩小以便提取更多的细节特征. 所以此类图像的最优 尺度应在原始尺度基础上往标号大的方向移动.

本文使用图像特征矩阵的稀疏度作为衡量图像内 容复杂度的依据. 图像特征矩阵是指在利用朝向柱阵 列提取出图像的朝向特征后，得到的包含朝向、朝向 强度及位置信息的二维矩阵, 记为 $\mathbf{x}$. 利用 $\mathrm{L} 1$ 范数与 $\mathrm{L} 2$ 范数差异度来衡量特征矩阵 $\mathbf{x}$ 的稀疏度, 定义如下 ${ }^{[21]}$.

$s p(\mathbf{x})=\frac{\sqrt{K}-\left(\sum_{i=1}^{K}\left|x_{i}\right|\right) / \sqrt{\sum_{i=1}^{K} x_{i}{ }^{2}}}{\sqrt{K}-1}$,

其中, $K$ 为特征矩阵 $\mathbf{x}$ 包含的元素数目, $x_{i}$ 为特征矩阵拉 成向量后的第 $i$ 个元素. 依据上式得到图像在原始尺度 下朝向矩阵的稀疏度 $s p . s p$ 越大, 表示 $\mathbf{x}$ 越稀疏, 包含信 息量越少, 对应的图形越简单.

当 $s p$ 值小于阈值 $\theta$ 时, 认为原始尺度的图像足够复 杂，该尺度下的特征矩阵可以表征该图像的足够信息， 此时可将最优尺度 $n_{\mathrm{opt}}$ 设置为原始尺度, 即 $n_{\mathrm{opt}}=n_{1}$; 当 $s p \geq \theta$ 时, 则认为该图像在原始尺度下过于稀疏，需要 对最优尺度进行调整. 最优尺度的标号与 $s p$ 的关系设 定如下:

$n_{\mathrm{opt}}= \begin{cases}1, & s p<\theta, \\ \lceil s p \times N\rceil, & s p \geq \theta,\end{cases}$
其中, 「. 表示向上取整操作. 由于 $s p$ 值的范围为 $[0,1]$, 所以 $n_{\mathrm{opt}} \in\{1,2, \ldots, N\}$. 当 $s p<\theta$ 时, $n_{\mathrm{opt}}$ 取原始尺度(标号 为 1$)$; 当图像过于稀疏时, $n_{\mathrm{opt}}$ 的标号随着稀疏度的增 加而增加.

与经典融合策略相同, 只有最优尺度以及比最优 尺度标号大的尺度参与融合, 且融合权重随着标号增 加而线性递减. 图3(b)给出了最优尺度融合策略下权 重曲线，其可看作图3(a)的经典权重曲线沿横轴方向 上的平移. 当 $s p<\theta$ 时, 曲线与经典融合策略中的曲线 相同. 在实际应用中, 为了增强结果稳定性, 可将与 $n_{\mathrm{opt}}$ 相邻的若干尺度均设置为最优尺度, 使用相同的最 大权重. 在本文中 $\theta$ 取 $1 / 2$.

确定各尺度的融合权重后，依据式(7)对各尺度下 获得的相似度集合进行融合. 具体过程如下.

设形状 $r$ 在尺度 $n$ 下返回的相似度集合构成的列向 量为 $\mathbf{s}_{r, n}$, 则形状 $r$ 在所有尺度下的相似度矩阵为 $\mathbf{S}_{r}=$ $\left(\mathbf{S}_{r, 1}, \ldots, \mathbf{S}_{r, n}, \ldots, \mathbf{S}_{r, N}\right)^{\mathrm{T}}$, 并进行如下计算得到尺度融合 后的相似度结果:

$\rho_{r}=\mathbf{w}_{r} \cdot \mathbf{S}_{r}$,

其中, $\mathbf{w}_{r}$ 是形状 $r$ 在所有尺度下的权重构成的行向量. 所有形状在多尺度融合后的相似度矩阵为

$\mathbf{S}=\left(\rho_{1}{ }^{\mathrm{T}}, \ldots, \rho_{r}{ }^{\mathrm{T}}, \ldots, \rho_{M}^{\mathrm{T}}\right)^{\mathrm{T}}$,

其中, $M$ 为形状总数目, $r \in\{1,2, \ldots, M\}$.

\section{3 实验过程与结果分析}

为了验证本文提出的最优尺度融合的线条上下文 (line context with optimized multi-scale fusion, Lc_Omf) 算法及经典尺度融合的线条上下文(line context with classic multi-scale fusion, Lc_Cmf)算法提取特征的有 效性, 首先将本文算法与 $\mathrm{LC}^{[16]} 、 \mathrm{Hu}$ 矩 ${ }^{[7]}$ Z Zernike 矩 ${ }^{[8]} 、 \mathrm{SC}^{[3]} 、 \mathrm{FD}^{[6]}$ 和 $\mathrm{Sift}^{[23]}$ 等传统算法在衡量形状描 述子可靠性的基准数据库Kimia数据集 ${ }^{[24]}$ 上进行了检 索正确率的对比, 其次对比分析了最优融合策略相对 于传统尺度融合策略的优势, 最后在遮挡和加噪声数 据集上测试了算法的鲁棒性. 本文算法均表现出明显 优势.

其中Kimia99数据库由 9 类图像组成, 每类有 11 个 形状, 共 99 个形状. Kimia216数据库由 18 类图像组成, 
每类有 12 个形状，共 216 个形状. 检索结果的评价采用 Bull-eye度量方法 ${ }^{[25]}$.

\section{1 特征表示能力的比较}

如表2所示, Lc_Omf在Kimia216与Kimia99数据集 上取得了更好的检索结果. 实验中的参数设置如下: Lc_Cmf选取 $N=12$ 个尺度, 2 级朝向强度; Lc_Omf融合 $N=18$ 个尺度中的 3 个最优尺度, 2 级朝向强度.

\section{2 融合策略的对比}

对Kimia216与Kimia99数据集中各类形状在优化 选择前后的结果进行差异性比较, 结果如图4所示. 图 中给出了Lc_Omf和Lc_Cmf在每一类上检索正确率差 值的统计直方图，横轴数值为 Lc_Omf正确率减 Lc_Cmf正确率的差值, 差值为正(直方图位于第一象 限)表明Lc_Omf 效果好于Lc_Cmf. 由图可见，在所有 类上，采用最优融合策略都取得了比经典策略更高的 检索正确率.

表 2 Kimia数据集中不同算法的检索率

Table 2 Retrieval rates of different algorithms on Kimia dataset

\begin{tabular}{ccc}
\hline \multirow{2}{*}{ 算法 } & \multicolumn{2}{c}{ 检索率(\%) } \\
\cline { 2 - 3 } & Kimia99 & Kimia216 \\
\hline Zernike矩 & 62.54 & 67.03 \\
Hu矩 & 68.87 & 65.63 \\
LC & 60.97 & 73.07 \\
SC & 76.36 & 76.14 \\
Sift & 77.04 & 65.28 \\
FD & 82.19 & 80.29 \\
Lc_Cmf & 77.69 & 80.83 \\
Lc_Omf & $\mathbf{8 5 . 4 0}$ & $\mathbf{8 6 . 8 8}$ \\
\hline
\end{tabular}

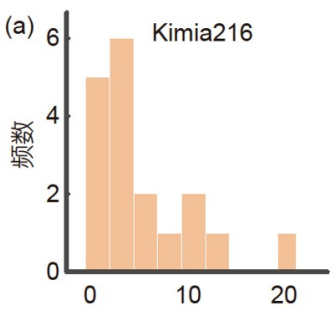

检索率差值(Lc_Omf-Lc_Cmf) (\%)

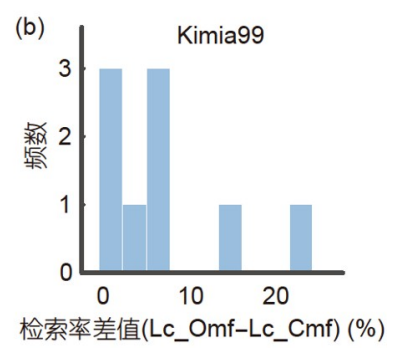

图 4 在各类上检索率差值的直方图

Figure 4 Histograms of retrieval-rate-difference on various classes.

\section{3 抗干扰实验}

为了验证本文算法在干扰环境下的性能，对 $\mathrm{Ki}$ mia216形状数据库中的形状添加遮挡, 对图像中含有 像素的 $10 \%$ $80 \%$ 的区域进行遮挡，构成 8 个不同遮挡 率的目标数据库, 如图 5 所示.

在遮挡数据集比较本文的Lc_Omf算法与其他算 法的检索正确率, 结果如图6所示. 可以看出, 随着遮挡 程度的增加, 所有算法的检索率均有一定下降, 但本文 算法下降较缓, 且在所有遮挡水平下均优于其他算法.

为了验证本文算法在噪声环境下的性能, 对 Kimia216形状数据库中的图像 $5 \%$ \% $50 \%$ 的区域添加椒盐 噪声, 噪声值的百分比称为噪声密度. 构成 10 个不同椒 盐噪声密度的目标数据库, 如图7所示.

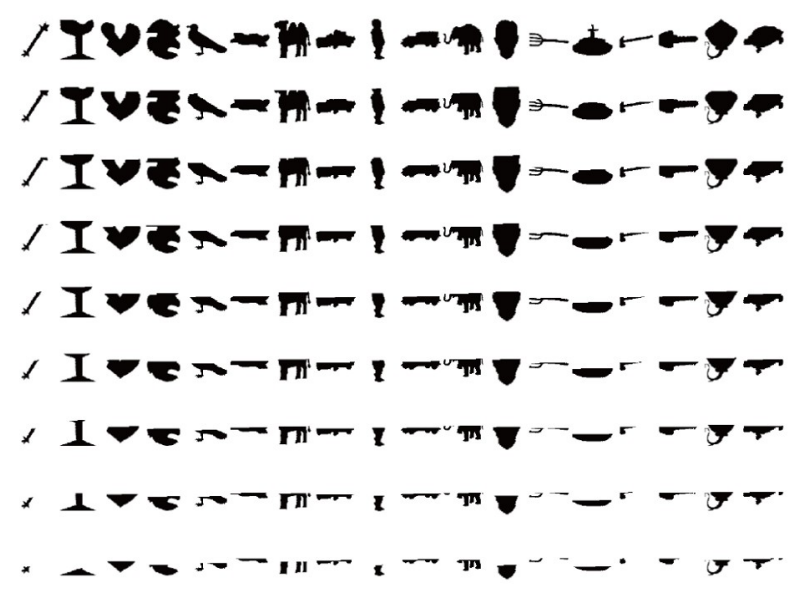

图 5 Kimia216数据集添加不同程度遮挡示意

Figure 5 Illustration of adding occlusion to the Kimia216 dataset.

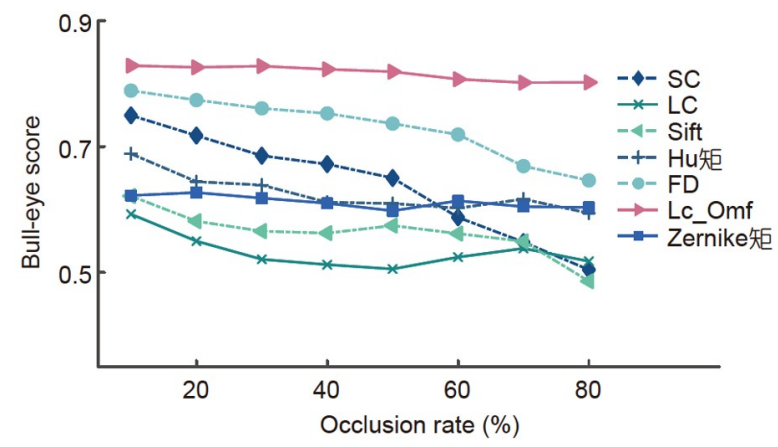

图 6 不同程度遮挡下的检索率曲线

Figure 6 Retrieval rate curve on datasets with different degrees of occlusion. 


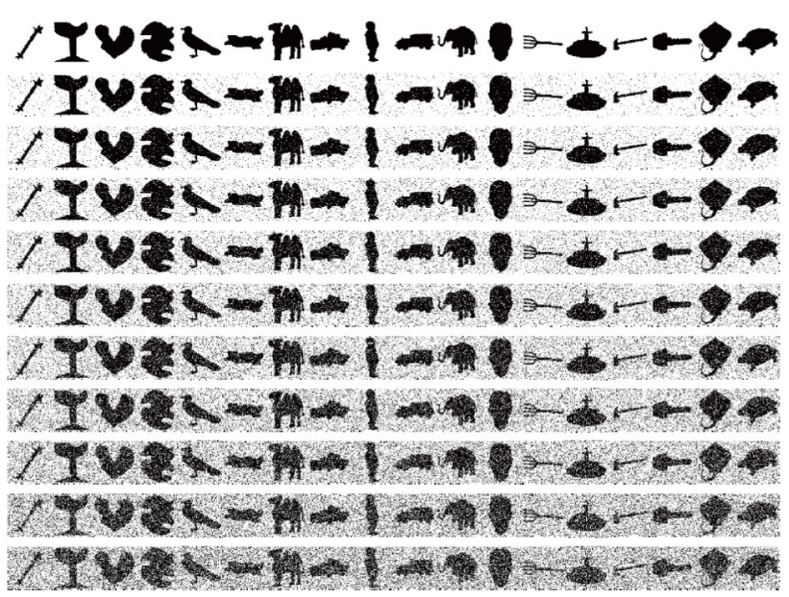

图 7 Kimia216数据集添加不同程度椒盐噪声示意

Figure 7 Illustration of adding salt-pepper noise to the Kimia216.

\section{在添加椒盐噪声的数据集比较本文的Lc_Omf算} 法与其他算法的检索正确率, 结果如图8所示. 可以看 出, 存在噪声干扰时, 本文算法仍然拥有较为优秀的特 征表示能力.

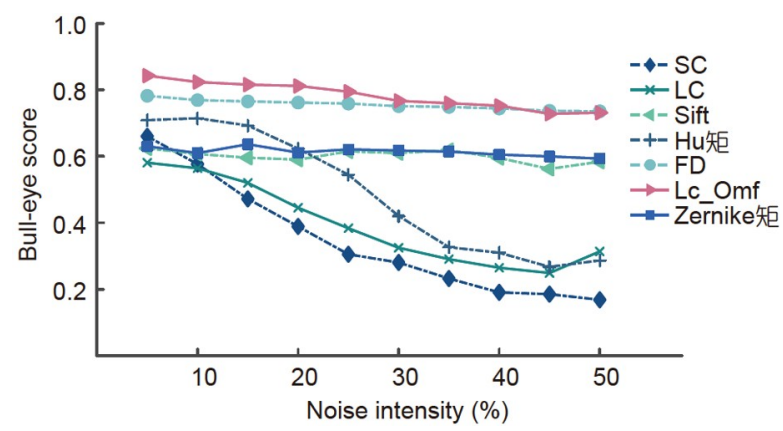

图 8 不同程度噪声干扰下的检索率曲线

Figure 8 Retrieval rate curve on datasets with different degrees of salt-pepper noise.

\section{4 结论}

本文提出的基于CRF朝向敏感的多尺度形状特征 表示方法通过引入朝向强度和多尺度信息，获得了较 强的图像形状特征表示能力. 实验结果表明本文算法, 尤其是使用了最优融合策略的特征表示方法可以实现 更高的检索精度，且在存在遮挡和噪声条件下表现出 优良的鲁棒性.

\section{参考文献}

1 Zheng X, Ji R, Chen Y, et al. MIGO-NAS: Towards fast and generalizable neural architecture search. IEEE Trans Pattern Anal Mach Intell, 2021, 43: 2936-2952

2 Tang X, Hao K, Wei H, et al. Using line segments to train multi-stream stacked autoencoders for image classification. Pattern Recognit Lett, 2017, 94: 55-61

3 Papaioannou A, Antonakos E, Zafeiriou S. Complex representations for learning statistical shape priors. In: 25th European Signal Processing Conference (EUSIPCO), 2017. 1180-1184

4 Alajlan N, Kamel M S, Freeman G H. Geometry-based image retrieval in binary image databases. IEEE Trans Pattern Anal Mach Intell, 2008, 30: $1003-1013$

5 Ribas L C, Neiva M B, Bruno O M. Distance transform network for shape analysis. Inf Sci, 2019, 470: 28-42

6 López-Ávila L F, Álvarez-Borrego J, Solorza-Calderón S. Fractional Fourier-radial transform for digital image recognition. J Sign Process Syst, 2021, 93: 49-66

7 Hjouji A, EL-Mekkaoui J, Jourhmane M. Rotation scaling and translation invariants by a remediation of Hu's invariant moments. Multimed Tools Appl, 2020, 79: 14225-14263

8 Singh C, Aggarwal A. An effective approach for noise robust and rotation invariant handwritten character recognition using Zernike moments features and optimal similarity measure. Appl Artif Intell, 2020, 34: 1011-1037

9 Ling H, Jacobs D W. Shape classification using the inner-distance. IEEE Trans Pattern Anal Mach Intell, 2007, 29: 286-299

10 Wang J, Bai X, You X, et al. Shape matching and classification using height functions. Pattern Recognit Lett, 2012, 33: 134-143

11 de Haan E, Aleman A. Mental imagery: In search of a theory. Behav Brain Sci, 2002, 25: 188-189

12 Yan D, Li K, Gu S, et al. Network-based bag-of-words model for text classification. IEEE Access, 2020, 8: 82641-82652

13 Xiao R, Wang J, Guo X, et al. Open curvature scale space matching for coronary artery identification in X-ray angiographic images. IEEE Access, 2020, 8: 16989-17001 
$14 \mathrm{Hu}$ R, Jia W, Ling H, et al. Angular pattern and binary angular pattern for shape retrieval. IEEE Trans Image Process, 2014, 23: 1118-1127

15 Wang X, Feng B, Bai X, et al. Bag of contour fragments for robust shape classification. Pattern Recognit, 2014, 47: 2116-2125

16 Wei H, Li Q, Dong Z. Learning and representing object shape through an array of orientation columns. IEEE Trans Neural Netw Learn Syst, 2014, 25: 1346-1358

17 Wei H, Dong Z, Liu B. Hypercolumn-array based image representation and its application to shape-based object detection. Appl Soft Comput, 2017, 52: 333-347

18 Bosking W H, Crowley J C, Fitzpatrick D. Spatial coding of position and orientation in primary visual cortex. Nat Neurosci, 2002, 5: 874-882

19 Zhou W. Research on multiscale shape description and retrieval algorithm (in Chinese). Dissertation for Master's Degree. Suzhou: Soochow University, 2020 [周雯. 形状的多尺度描述与检索算法研究. 硕士学位论文. 苏州: 苏州大学, 2020]

20 Wang F, Yang W N, Wang J, et al. Selection and evaluation of the optimal scale in multiscale segmentation of remote sensing images (in Chinese). Remote Sens Tech Appl, 2020, 35: 623-633 [王芳, 杨武年, 王建, 等. 遥感影像多尺度分割中最优尺度的选取及评价. 遥感技术与 应用, 2020, 35: 623-633]

21 Hoyer P O. Non-negative matrix factorization with sparseness constraints. J Mach Learn Res, 2004, 5: 1457-1469

22 Li X. The scale optimal selection for image global representation (in Chinese). Dissertation for Master's Degree. Harbin: Harbin Institute of Technology, 2013 [李雪. 面向全局图像表达的尺度优化选择. 硕士学位论文. 哈尔滨: 哈尔滨工业大学, 2013]

23 Wang Y, Zhang X. Digital core image registration based on SIFT features. J Phys-Conf Ser, 2021, 1748: 042047

24 Sebastian T B, Klein P N, Kimia B B. Recognition of shapes by editing their shock graphs. IEEE Trans Pattern Anal Mach Intell, 2004, 26: 550571

25 Liao N, Guo B, Li Z, et al. An advanced Fourier descriptor based on centroid contour distances. J Phys-Conf Ser, 2021, 1735: 012002

\title{
Shape feature representation by the multiscale model of CRF orientation selectivity
}

\author{
DUAN Ting, LI Ming, SHEN Hui \& HU DeWen \\ College of Intelligence Science and Technology, National University of Defense Technology, Changsha 410073, China
}

As a stable feature of the objects in images, the contour is one of the bases of invariant recognition, such as rotation, translation, and scale. Inspired by the orientation selectivity in the primary visual cortex, this paper extracts the orientation intensities in images by modeling the receptive field of visual neurons and proposes a shape feature representation strategy similar to brain perception. First, to obtain abundant contour information, the primitive geometric features of the object are represented by the orientation intensity of line segments at multiple scales. Second, we improve the orientation matching and multiscale fusion strategy by using the sparsity index. We also obtain more representative shape features. The experimental results suggest that the shape retrieval algorithm based on our feature representation strategy achieves a higher retrieval accuracy and stronger robustness in the presence of occlusion and noise.

\section{shape feature representation, multiscale fusion, orientation selectivity, geometric feature extraction, image} representation

doi: 10.1360/SST-2021-0226 